\title{
Atmospheric correction of HJ1-A/B images and the effects on remote sensing monitoring of cyanobacteria bloom
}

\author{
HONGXU MA, SHENGLIAN GUO, XINGJUN HONG \& YANLAI ZHOU \\ State Key Laboratory of Water Resources and Hydropower Engineering Science, Wuhan University, Wuhan, Hubei \\ Province, 430072, China \\ mahongxu@whu.edu.cn
}

\begin{abstract}
The HJ-1A/B satellite offers free images with high spatial and temporal resolution, which are effective for dynamically monitoring cyanobacteria blooms. However, the HJ-1A/B satellite also receives distorted signals due to the influence of atmosphere. To acquire accurate information about cyanobacteria blooms, atmospheric correction is needed. HJ-1A/B images were atmosphere corrected using the FLAASH atmospheric correction model. Considering the quantum effect within a certain wavelength range, a spectral response function was included in the process. Then the model was used to process $\mathrm{HJ}-1 \mathrm{~A} / \mathrm{B}$ images, and the NDVI after atmospheric correction was compared with that before correction. The standard deviation improved from 0.13 to 0.158 . Results indicate that atmospheric correction effectively reduces the distorted signals. Finally, NDVI was utilized to monitor the cyanobacteria bloom in Donghu Lake. The accuracy was enhanced compared with that before correction.
\end{abstract}

Key words atmospheric correction; cyanobacteria monitoring; NDVI; FLAASH

\section{INTRODUCTION}

Remote sensing has a great advantage of macroscopic, rapid and inexpensive monitoring of the environment (Li et al., 2011). An increasing amount of research has developed in ecological environment monitoring. Prangsma and Roozekrans (1989) using remote sensing images successfully recognized cyanobacteria bloom for first time. Kuster (2004), using a biological optical mode monitored cyanobacteria bloom in the Baltic Sea. Chen and Dai (2008) extracted cyanobacteria bloom information using CBERS images and the CART method that determined the threshold. Duan et al. (2008) extracted cyanobacteria bloom information using MODIS/Terra, CBERS and IRS-P6images by comparing single band data with difference values and specific values. Wang et al. (2008) agreed that water temperature is one important factor of cyanobacteria bloom by studying MODIS images.

Sensors on the HJ-1A/B satellite receive the reflection of solar radiation signals from surface features. The signals distort and lose accuracy under the influence of the atmosphere and radiation. This distortion is more serious for cyanobacteria bloom monitoring, because the reflection from water is weaker than that from land. Researchers have proposed many models for atmospheric correction, such as the histogram equalization method, empirical line method and 6S. These methods are based on relatively ideal conditions, which lead to a limited accuracy. The influence will be more serious when monitoring cyanobacteria due to the relatively weak signals of water.

This paper describes research on Donghu Lake in Wuhan City. FLAASH is an atmospheric correction model, while MODTRAN is an atmospheric transmission model. Thus FLAASH, which is based on the MODTRAN atmospheric transmission model was used to process atmospheric correction for $\mathrm{HJ}-1 \mathrm{~A} / \mathrm{B}$ data. The spectral respond function was introduced and a weighted average calculated into the process, considering the influence of the quantum effect in a certain range. By comparing the NDVI data before and after atmospheric correction, the effect of FLAASH was valued. Then this method was used to extract cyanobacteria information and to compare the impact on accuracy before and after FLAASH atmospheric correction.

\section{ATMOSPHERIC CORRECTION PROCESS}

\section{Radiation correction}

Raw remote sensing images express the value by digital number (DN). DN needs to be calculated into radiation values to obtain the real reflectance of surface features. Remote sensing satellites are 
radiometric calibrated before launch, and repeatedly recalibrated. The radiation correction of $\mathrm{HJ}-1 \mathrm{~A} / \mathrm{B}$ is:

$$
L=\frac{D N}{a}+L_{0}
$$

where $L$ represents radiation values, $D N$ represents the digital number, $a$ represents scales, $L_{0}$ represents offset. The units of $L$ are $\mathrm{W} \mathrm{m}^{-2} \mathrm{sr}^{-1} \mu \mathrm{m}^{-1}$.

\section{FLAASH atmospheric correction model}

FLASSH was developed by SSI, AFRL and SITAC, based on the Moderate resolution atmospheric transmission and Beer-Lambert surface model (Peng et al., 2007):

$$
L=\left[\frac{A \rho}{1-\rho_{e} s}\right]+\left[\frac{B \rho}{1-\rho_{e} s}\right]+L_{a}
$$

where $L$ represents total radiation received by sensors. $\rho$ is pixel reflectivity. $\rho_{e}$ is average reflectivity in the region, $S$ is atmospheric sphere reflectivity, $L_{a}$ is atmospheric backscatter radiation, and $A$ and $B$ are coefficients depending on atmospheric conditions.

The first item of the formula represents the pixel reflectivity which enters the sensor directly. The second item represents the reflectivity from surface features through atmospheric scattering. The third represents solar radiation which enters the atmosphere through atmospheric scattering and then enter into sensors.

FLAASH calculates $A, B, S$ and $L_{a}$ by using water vapour in the air and aerosol optical thickness. FLAASH records the inverse optical thickness of an aerosol by the black object method. MODTRAN used the results of simulation in several different conditions to calculate a look-up table for water vapour.

FLAASH corrects for the effect of neighbouring points by atmospheric point spread methods, (Li et al., 2008):

$$
L_{e} \approx\left[\frac{(A+B) \rho_{e}}{1-\rho_{e} s}\right]+L_{a}
$$

where $L_{e}$ represents the spatial average of the centre points and neighbouring points.

\section{Parameters of FLAASH atmospheric correction}

The FLAASH atmospheric correction model has several important parameters, such as longitude and latitude of focus, altitude of sensor, size of pixels, average altitude of the range, local time, an atmospheric model, aerosol model and aerosol inversion method. The spectrum response function of HJ-1A/B is also important here. The altitude of the sensor and the size of pixels can be obtained on the HJ-1A/B website. The longitude and latitude of focus and local time can be obtained in the head file of $\mathrm{HJ}-1 \mathrm{~A} / \mathrm{B}$ raw files. The average altitude of the range can be calculated by using a local DEM. Here, due to the lack of measured data, we use a standard empirical model and mid-latitude winter model as the atmospheric model, an urban model and an aerosol model, and use 2-B(K-T) as the aerosol inversion method.

The spectral response function is also introduced in this method. Every band has a certain range, such as HJ-1A CCD which uses $430-520 \mathrm{~nm}$ to represent its Band 1. However, there is a remarkable difference among every point of the photosensitive elements of the sensor. The midvalue represents the whole band in normal calculations. The accuracy will significantly improve by introducing the spectral response function and calculating the weighted average value of every signal in the whole band. This method takes the quantum effect out of a certain range into consideration to enhance the accuracy of atmospheric correction.

The calculated spectral response function of HJ-1A CCD2 is shown in Fig. 1; from left to right the bands are: $0.43-0.52 \mathrm{~nm}, 0.52-0.6 \mathrm{~nm}, 0.63-0.69 \mathrm{~nm}$ and $0.76-0.9 \mathrm{~nm}$. 


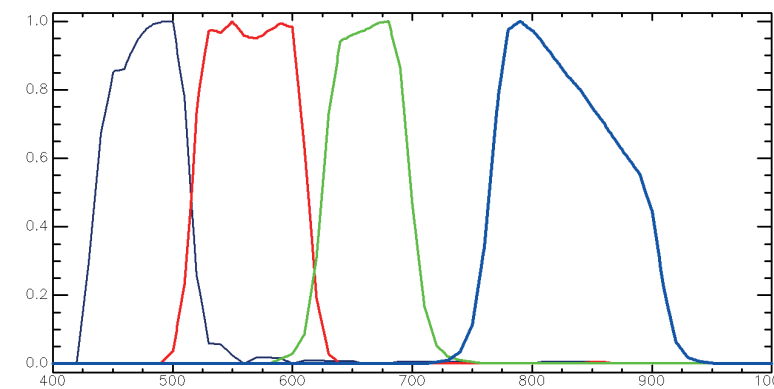

Fig. 1 Spectral response function of HJ-1A CCD2.

\section{Evaluation index}

After FLAASH atmospheric correction, the images' contrast ratio will increase and the information will be enriched. The difference between water and non-water will increase and help monitoring of cyanobacteria bloom. The NDVI index is used to evaluate the effect of atmospheric correction. The formula is shown as follows:

$$
N D V I=\frac{N I R-R E D}{N I R+R E D}
$$

where NIR represents the near-infrared band and RED represents the red band.

The NDVI for both images were calculated before and after atmospheric correction and the statistics of NDVI values for the target region. The variation of the mean value will reflect the change in luminance. The variation of the standard deviation value will reflect the change in volume of land surface information. The higher the standard deviation, the more the range of grey value and the more information is enhanced in the images.

\section{STUDY CASE}

\section{Study region}

Donghu Lake is located in the northeast of Wuchang District, Wuhan City. The area of the Lake is $27.9 \mathrm{~km}^{2}$, the average depth is $2.21 \mathrm{~m}$ and the deepest part is $6 \mathrm{~m}$. There are more than 120 islands in this region. The major problem of Donghu Lake is eutrophication, which will lead to a huge amount of reproduction of cyanobacteria. The cyanobacteria bloom will lead to much damage to landscape and ecological system, and even endanger the drinking water security. As Donghu Lake is the largest inner-city lake, the cyanobacteria bloom will affect the city more seriously than a lake out of the city. In this situation, it is significant for the ecological system of Wuhan city to do research on how to monitor cyanobacteria bloom.

\section{Comparison of water information before and after atmospheric correction}

During the process of radiance correction, different sensors with different bands have different radiance correction parameters. In this paper, HJ-1A CCD2 images were processed and the parameters are shown in Table 1. The images were taken on 17 November 2013 and the parameters of FLAASH are shown in Table 2. A comparison of water information before and after FLAASH atmospheric correction is shown in Fig. 2.

As shown Fig. 3, the tendency of NDVI stays the same. However, the range of NDVI has enlarged after the atmospheric correction, which offers data with more information for cyanobacteria monitoring. This paper picked 791 points to compare and calculate the statistics. The variation of the maximum and the minimum shows the degree of enhancement. Data and the Box plot are shown in Table 3. 
Table 1 Coefficients of radiometric correction of HJ-1A CCD2 on 17 November $2013\left(\mathrm{~W} \mathrm{~m}^{-2} \mathrm{sr}^{-1} \mu \mathrm{m}^{-1}\right)$.

\begin{tabular}{lllll}
\hline Band & Band 1 & Band 2 & Band 3 & Band 4 \\
\hline$\alpha$ & 0.7435 & 0.7379 & 1.0899 & 1.0852 \\
$L_{0}$ & 4.6344 & 4.0982 & 3.7360 & 0.7385 \\
\hline
\end{tabular}

Table 2 Parameters of FLAASH atmospheric correction.

\begin{tabular}{lllll}
\hline Date & Local time & Sensor altitude & Solar altitude & Solar azimuth \\
\hline 2013-11-17 & $11: 06: 08$ & $650 \mathrm{~km}$ & $30.707^{\circ}$ & $321.692^{\circ}$ \\
\hline Atmospheric Model & Aerosol model & Longitude & Latitude & Altitude \\
\hline Mid-latitude Winter & Urban & $114.28472^{\circ}$ & $29.84728^{\circ}$ & $53 \mathrm{~m}$ \\
\hline
\end{tabular}

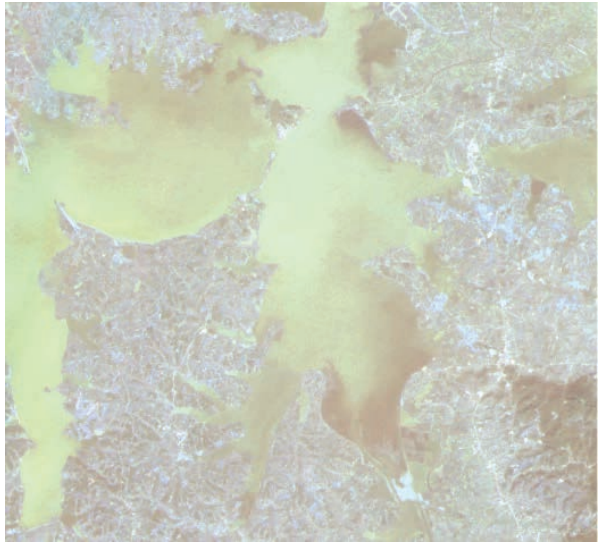

(a)

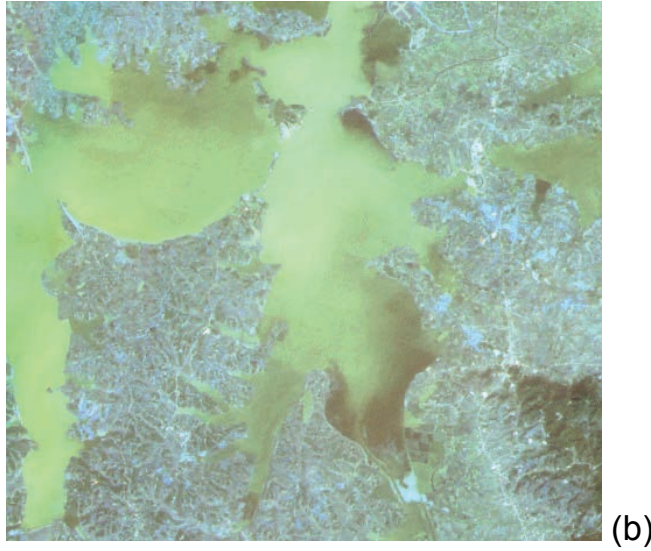

(b)

Fig. 2 Result of comparison of before and after FLAASH atmospheric correction; (a) before atmospheric correction, and (b) after atmospheric correction.

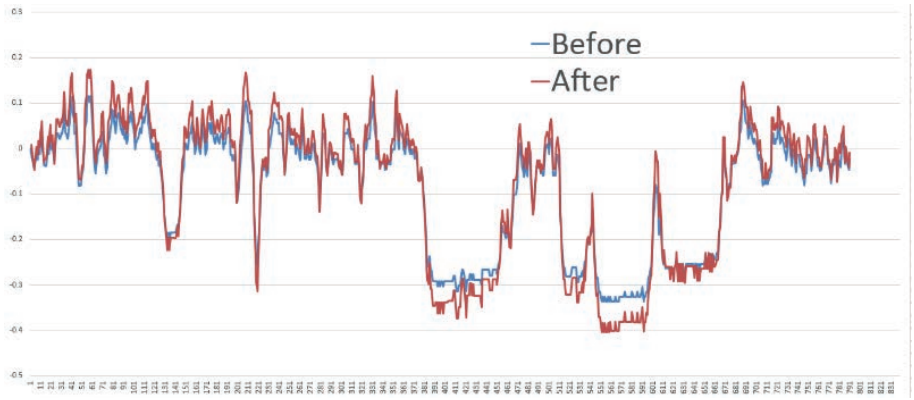

Fig. 3 NDVI Comparison between before and after FLAASH atmospheric correction

Table 3 Data of NDVI comparison between before and after atmospheric correction.

\begin{tabular}{lllll}
\hline & Mean & Maximum & Minimum & Standard deviation \\
\hline Before & -0.08906 & 0.115434 & -0.33639 & 0.13052 \\
After & -0.08063 & 0.173284 & -0.40415 & 0.15786 \\
\hline
\end{tabular}

\section{Comparison of cyanobacteria monitoring before and after atmospheric correction}

A high density of cyanobacteria has a steep slope effect like that of the plant spectral curve in the near-infrared band, thus the NDVI index can be used to extract cyanobacteria information. As a ratio type function, the NDVI index can eliminate the error effects of solar altitude, terrain and observation angle. As a normalization type function, the NDVI index can also eliminate the error of damping of the sensor. The NDVI index is a significant method for describing the growth 
situation and spatial distribution of plants, which is why NDVI is used widely in monitoring the environment in inland lakes widely.

The information of the images was enhanced after atmospheric correction, and the radiance difference between consecutive pixels, which makes a significant contribution to extracting the cyanobacteria information. The comparison of NDVI before and after FLAASH atmospheric correction is shown in Fig. 4.
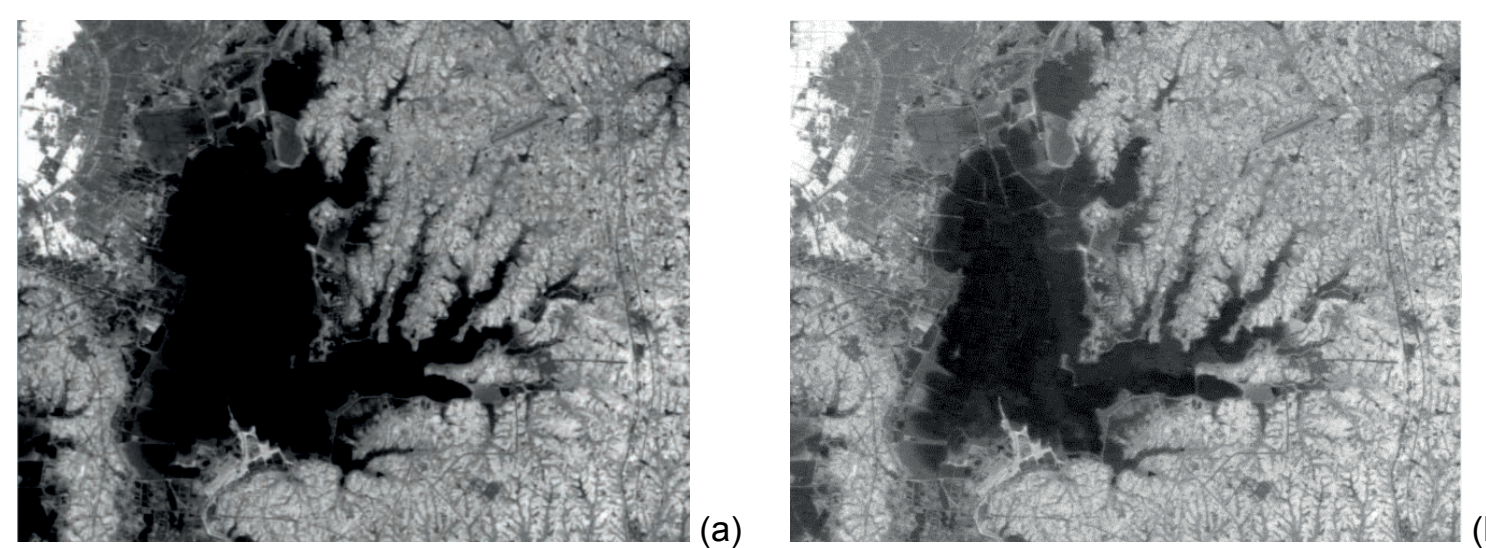

(b)

Fig. 4 NDVI comparison between (a) before and (b) after FLAASH atmospheric correction.

The right image (Fig. 4) is the NDVI result after atmospheric correction. The paddy fields on the lakeside are enhanced successfully, which is ignored in the left image. After on-site scrutiny, the paddy fields were found to be filled with cyanobacteria due to their abandonment in November, which proved the validity of the FLAASH atmospheric correction and the accuracy of the NDVI index. Then this proved method was used to monitor the cyanobacteria bloom in Donghu Lake. The results are shown in Fig. 5.

The threshold segmentation method was used to determine the degree of cyanobacteria bloom. Green indicates the high concentration, red indicates the low concentration and the blue represents the dyke, road and other features.

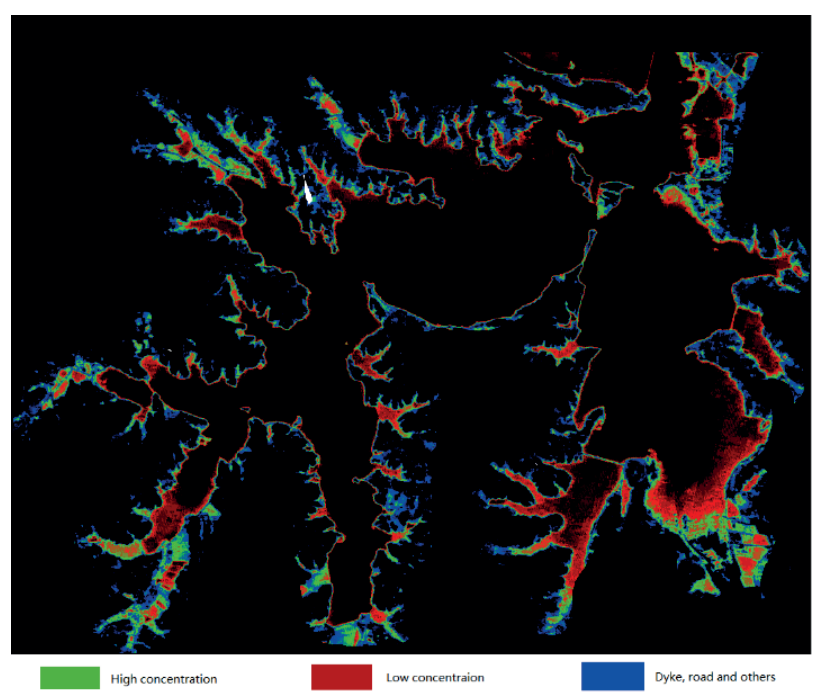

Fig. 5 Cyanobacteria bloom NDVI after FLAASH atmospheric correction.

\section{CONCLUSION}

The HJ-1A/B images have a relatively high spatial resolution and high time resolution compared with other free remote sensing images, which mean they can be used to monitor the environment 
efficiently and effectively. The FLAASH atmospheric correction model was used to process the HJ-1A/B images. Based on the quantum effect, the spectrum response function was introduced into the atmospheric correction model, and the results was highly improved. The comparison between results before and after atmospheric correction showed that the contrast between pixels was enhanced and the difference between water feature and non-water feature was also greater. The NDVI index showed that the wave crest and the wave hollow were enhanced. The paddy fields and the cyanobacteria in the fields were recognized effectively. Then using this method to calculate the NDVI index of Donghu Lake, the cyanobacteria bloom information was monitored. A threshold segmentation method was used to determine the degree of cyanobacteria bloom, and separated high concentration from low concentration efficiently. This method offers convincing information for cyanobacteria bloom monitoring.

\section{REFERENCES}

Chen Liqiong, et al. (2011) Water color constituents remote sensing in Wuhan Donghu Lake using HJ-1A/B CCD imagery. Geomatics and Information Science of Wuhan University 36(11), 1280-1283 (in Chinese).

Chen Yun and Dai Jinfang (2008) Extraction methods of cyanobacteria bloom in Lake Taihu based on RS data. Journal of Lake Sciences 20(2), 179-183 (in Chinese).

Duan Hongtao, Zhang Shouxuan and Zhang Yuanzhi (2008) Cyanobacteria bloom monitoring with remote sensing in Lake Taihu. Journal of Lake Sciences 20(2), 145-152 (in Chinese).

Kutser, T. (2004) Quantitative detection of chlorophyll in cyanobacterial blooms by satellite remote sensing. Limnology and Oceanography 49(6), 2179-2189.

Li Yachun, et al. (2011) Extraction methods of cyanobacteria bloom in Lake Tai based on MODIS vegetation index. Journal of the Meteorological Sciences 31(6), 737-741 (in Chinese).

Li Guoyan, et al. (2008) Atmospheric correction of MODIS and its application in cyanobacteria bloom monitoring in Lake Taihu. Journal of Lake Sciences 20(2), 160-166 (in Chinese).

Peng Guangxiong, et al. (2007) Study on CBERS-2's CCD image cross calibration and atmospheric correction. Journal of Infrared Millim 26(1), 22-25 (in Chinese).

Prangsma, G.J. and Roozekrans, J.N. (1989) Using AVHRR HRPT imagery in assessing water quality parameters. International Journal of Remote Sensing 10(4-5), 811-818.

Wang Deyu, et al. (2008) Relationship between blue algal bloom and water temperature in Lake Taihu based on MODIS. Journal of Lake Sciences 20(2), 173-178 (in Chinese). 